\title{
Characterising metabolically healthy obesity in weight-discordant monozygotic twins
}

\author{
J. Naukkarinen • S. Heinonen • A. Hakkarainen • J. Lundbom • K. Vuolteenaho • \\ L. Saarinen • S. Hautaniemi • A. Rodriguez • G. Frühbeck • P. Pajunen • \\ T. Hyötyläinen • M. Orešič • E. Moilanen • A. Suomalainen • N. Lundbom • \\ J. Kaprio $\cdot$ A. Rissanen $\cdot$ K. H. Pietiläinen
}

Received: 10 August 2013 / Accepted: 4 September 2013 / Published online: 8 October 2013

(C) Springer-Verlag Berlin Heidelberg 2013

\begin{abstract}
Aims/hypothesis Not all obese individuals display the metabolic disturbances commonly associated with excess fat accumulation. Mechanisms maintaining this 'metabolically
\end{abstract}

Electronic supplementary material The online version of this article (doi:10.1007/s00125-013-3066-y) contains peer-reviewed but unedited supplementary material, which is available to authorised users.

J. Naukkarinen · S. Heinonen • A. Rissanen • K. H. Pietiläinen $(\triangle)$

Obesity Research Unit, Research Programs Unit, Diabetes and Obesity, University of Helsinki, Biomedicum Helsinki, C424b, PO Box 63, Helsinki, Finland 00014

e-mail: kirsi.pietilainen@helsinki.fi

J. Naukkarinen $\cdot$ J. Kaprio $\cdot$ K. H. Pietiläinen FIMM, Institute for Molecular Medicine Finland,

University of Helsinki, Helsinki, Finland

J. Naukkarinen

Public Health Genomics Unit, National Institute for Health and Welfare, Helsinki, Finland

A. Hakkarainen $\cdot$ J. Lundbom $\cdot$ N. Lundbom

Helsinki Medical Imaging Center, University of Helsinki,

Helsinki, Finland

K. Vuolteenaho $\cdot$ E. Moilanen

The Immunopharmacology Research Group, University of Tampere

School of Medicine and Tampere University Hospital,

Tampere, Finland

L. Saarinen $\cdot$ S. Hautaniemi

Computational Systems Biology Laboratory, Genome-Scale Biology

Research Program, Institute of Biomedicine, University of Helsinki,

Helsinki, Finland

\section{A. Rodriguez $\cdot$ G. Frühbeck}

Metabolic Research Laboratory, Clinica Univ. de Navarra,

University of Navarra, Pamplona, Spain healthy obesity' (MHO) are as yet unknown. We aimed to study different fat depots and transcriptional pathways in subcutaneous adipose tissue (SAT) as related to the MHO phenomenon.
A. Rodriguez $\cdot$ G. Frühbeck

Centro de Investigación Biomédica en Red de Fisiopatología de la Obesidad y Nutrición (CIBERobn)

URL: www.ciberobn.es

\section{P. Pajunen}

Diabetes Prevention Unit, Division of Welfare and Health Promotion, National Institute for Health and Welfare, Helsinki, Finland

T. Hyötyläinen • M. Orešič

Systems Biology and Bioinformatics, VTT Technical Research Centre of Finland, Espoo, Finland

\section{A. Suomalainen}

Research Program of Molecular Neurology and Department of Neurology, University of Helsinki and Helsinki University Central Hospital, Helsinki, Finland

\section{J. Kaprio}

Finnish Twin Cohort Study, Hjelt Institute, University of Helsinki, Helsinki, Finland

\section{J. Kaprio}

Department of Mental Health and Substance Abuse Services, National Institute for Health and Welfare, Helsinki, Finland

\section{K. H. Pietiläinen}

Division of Endocrinology, Department of Medicine, Helsinki University Central Hospital, Helsinki, Finland 
Methods Sixteen rare young adult obesity-discordant monozygotic (MZ) twin pairs (intra-pair difference $(\Delta)$ in BMI $\geq 3 \mathrm{~kg} / \mathrm{m}^{2}$ ), aged $22.8-35.8$ years, were examined for detailed characteristics of metabolic health (subcutaneous, intraabdominal and liver fat [magnetic resonance imaging/ spectroscopy]), OGTT, lipids, adipokines and C-reactive protein (CRP). Affymetrix U133 Plus 2.0 chips were used to analyse transcriptomics pathways related to mitochondrial function and inflammation in SAT.

Results Based on liver fat accumulation, two metabolically different subgroups emerged. In half $(8 / 16)$ of the pairs ( $\Delta$ weight $17.1 \pm 2.0 \mathrm{~kg}$ ), the obese co-twin had significantly higher liver fat $(\Delta 718 \%), 78 \%$ increase in AUC insulin during OGTT and CRP, significantly more disturbance in the lipid profile and greater tendency for hypertension compared with the lean co-twin. In these obese co-twins, SAT expression of mitochondrial oxidative phosphorylation, branched-chain amino acid catabolism, fatty acid oxidation and adipocyte differentiation pathways were downregulated and chronic inflammation upregulated. In the other eight pairs $(\Delta$ weight $17.4 \pm 2.8 \mathrm{~kg}$ ), the obese co-twin did not differ from the nonobese co-twin in liver fat $(\Delta 8 \%)$, insulin sensitivity, CRP, lipids, blood pressure or SAT transcriptomics.

Conclusions/interpretation Our results suggest that maintenance of high mitochondrial transcription and lack of inflammation in SAT are associated with low liver fat and MHO.

Keywords Adipose tissue - Diabetes · Fatty liver . Inflammation $\cdot$ Metabolically healthy obesity $\cdot$ Obesity

$\begin{array}{ll}\text { Abbreviations } \\ \text { BCAA } & \text { Branched-chain amino acid } \\ \text { CIRP } & \text { Chronic inflammatory response pathway } \\ \text { CRP } & \text { C-reactive protein } \\ \text { DXA } & \text { Dual energy x-ray absorptiometry } \\ \text { GCRMA } & \text { GeneChip robust multiarray averaging } \\ \text { GO } & \text { Gene ontology } \\ \text { hsCRP } & \text { High-sensitivity CRP } \\ \text { MHO } & \text { Metabolically healthy obesity } \\ \text { MRS } & \text { Magnetic resonance spectroscopy } \\ \text { mtDNA } & \text { Mitochondrial DNA } \\ \text { MZ } & \text { Monozygotic } \\ \text { SAT } & \text { Subcutaneous adipose tissue } \\ \text { VAT } & \text { Visceral adipose tissue }\end{array}$

\section{Introduction}

Obesity is associated with a cluster of metabolic disturbances including insulin resistance, dyslipidaemia and high blood pressure. A sizable subgroup (10-40\%) of obese individuals, however, seems to be protected from these disturbances [1-4].
The mechanisms behind the remarkably different outcomes of obesity are not yet well understood. Many [5-11] but not all [12] cross-sectional studies have shown that the metabolically healthier, insulin-sensitive obese individuals have less visceral adipose tissue (VAT), and less ectopic fat deposition in the liver [12] and skeletal muscle [7, 12, 13] than insulin-resistant obese individuals. A few studies also report that fatty liver remains the main correlate of insulin resistance independent of overall fat, VAT [14] and intramyocellular fat [15]. Such data suggest that low liver fat is a hallmark of metabolically more benign obesity. Interestingly, the proportion of the obese population without excess liver fat [16] is in the same range as that observed for metabolically healthy obesity (MHO) $[1,2]$.

It has been hypothesised that ectopic fat accumulation is caused by an inadequate subcutaneous adipose tissue (SAT) enlargement [17]. Indeed, lipodystrophic patients with total or partial absence of SAT have severe ectopic fat accumulation and insulin resistance [18]. In obesity, depression of SAT lipid storage after meals is related to abdominal obesity [19]. Furthermore, in normal-weight men, defective expression of lipid storage genes in SAT (DGAT2, SREBPIc [also known as SREBF1] and $C I D E A$ ) favours VAT accumulation during prolonged overfeeding [20]. These data suggest that handling of incoming energy in SAT is a key process in the prevention of VAT and perhaps also of ectopic fat.

Mitochondria, the organelles responsible for harvesting energy from food, are essential in the adipocyte differentiation programme [21]. Within human adipose tissue, mitochondrial DNA (mtDNA) copy number correlates with basal and insulin-stimulated lipogenesis [22, 23], suggesting that the enlargement of SAT is dependent on mitochondrial activity. We have earlier shown in obese (as compared with lean) monozygotic (MZ) co-twins that SAT mtDNA copy number and mitochondrial gene expression, especially branched-chain amino acid (BCAA) catabolism [24] and oxidative phosphorylation [25] pathways, are significantly downregulated in obesity. Low BCAA catabolism in SAT correlated with increased liver fat, whole-body insulin resistance and an inflammatory transcription profile in SAT [24]. Increased expression of inflammatory markers in SAT have previously been found to characterise obese women with high liver fat content compared with equally obese women with normal liver fat [26]. While definite causation cannot be claimed, the data suggest that inflammation and mitochondrial dysfunction in SAT underlie the development of the fatty liver. However, whether or not remaining free of these pathologies characterises $\mathrm{MHO}$ has not yet been studied.

Genetic and multiple environmental factors, as well as age, sex and age of onset of weight gain, may play a role in an individual's metabolic response to obesity. In the present study, we used a rare MZ obesity-discordant twin-pair design, which uniquely enables the control for genetic background, early development and environment, age and sex between 
obese and lean individuals [27]. In this contemporary collection of MZ twin pairs, we set out to study the relative contributions of different fat depots and their characteristics in relation to the development of obesity-associated pathological sequelae and insulin resistance. Concordant twin pairs were also included in the study for comparison purposes and to provide a wider distribution of within-pair differences in BMI.

\section{Methods}

Participants This study included 16 obesity-discordant (within-pair difference $[\Delta]$ in $\mathrm{BMI} \geq 3 \mathrm{~kg} / \mathrm{m}^{2}$ ) and 11 concordant MZ pairs $\left(\triangle B M I<3 \mathrm{~kg} / \mathrm{m}^{2}\right)$, identified from ten full birth cohorts of Finnish twins $(n=5,417$ families) [28]. Detailed description of the twins has been published previously [29]. In short, all pairs were white, with a mean age of 28.9 years (range 22.8-35.8 years). One obese co-twin had type 2 diabetes and used metformin and insulin. Another obese co-twin had inactive ulcerative colitis and used mesalazine and azathioprine. All other participants were healthy and normotensive and did not take any medications except for oral contraceptives. The study protocols were approved by the ethical committee of the Hospital District of Helsinki and Uusimaa, Finland. Written informed consent was obtained from all participants.

Body composition Weight, height, whole-body fat (dual energy x-ray absorptiometry [DXA]), abdominal subcutaneous and intra-abdominal fat (MRI) and liver fat (magnetic resonance spectroscopy [MRS]) were measured as described previously [29].

Blood tests and OGTT A $75 \mathrm{~g}$ OGTT was performed, after participants had fasted for $12 \mathrm{~h}$ overnight, to calculate HOMA-IR [30], Matsuda index [31] and insulinogenic index [32]. The twin with type 2 diabetes was not included in the OGTT analyses. Plasma glucose was measured using the spectrophotometric hexokinase and glucose-6-phosphate dehydrogenase (Roche Diagnostics, Basel, Switzerland) and serum insulin with time-resolved immunofluorometric assay (Perkin Elmer, Waltham, MA, USA). Additionally, fasting samples were used for determination of serum highsensitivity C-reactive protein (hsCRP) (Cobas CRP[Latex]HS; Roche Diagnostics), plasma leptin and adiponectin (DuoSet ELISA; R\&D Systems Europe, Abingdon, UK), serum total and HDL-cholesterol and triacylglycerol (Roche Diagnostics) and calculation of LDL-cholesterol by the Friedewald formula. The amino acids were analysed with Leco Pegasus 4D GC $\times$ GC-TOFMS instrument (Leco, St Joseph, MI, USA) as described previously [33]. Supine blood pressure was measured using a sphygmomanometer (mean of three measurements).
SAT analyses Biopsies of periumbilical SAT were obtained under local anaesthesia by a surgical technique and snapfrozen in liquid nitrogen for the extraction of total RNA (RNeasy Lipid Mini Kit; Qiagen, Venlo, the Netherlands) in 15 discordant and 11 concordant pairs. Transcriptomics analyses were performed by the Affymetrix U133 Plus 2.0 chips. The raw expression data were normalised using the GeneChip robust multiarray averaging algorithm and analysed using the GeneSpring GX 7.3 software (Agilent Technologies, Santa Clara, CA, USA). Based on the GO enrichment analysis and prior results, six pathways of interest - 'branched-chain amino acid (BCAA) catabolism' (gene ontology [GO]: 009083), 'fatty acid $\beta$ oxidation' (GO: 0006635), 'oxidative phosphorylation' (GO: 0006119), 'triacylglycerol synthesis' (GO: 0019432), 'white adipose cell differentiation' (GO: 0050872) and 'chronic inflammatory response, CIRP' (GO: 0002544) were selected, for which a mean centroid value (a geometric mean of the pathway) was constructed to represent an average activity of each of the pathways [24]. Details of the pathways and their constituents can be found in ESM Table 1. Individual transcript levels were used for leptin (probe 207092_at), adiponectin (207175_at) and hormone-sensitive lipase (LIPE) (208186_s_at). Transcript levels of leptin, adiponectin, LIPE and $M C P 1$ (also known as CCL2) were validated by quantitative PCR (iQ5 Real-Time PCR Detection System; Bio-Rad, Hercules, CA, USA) in 14 discordant pairs. Samples were run in triplicate and the comparative $\mathrm{Ct}$ method was applied using four housekeeping genes (B2M, YWHAZ, PPIA and RPLPO) (primers available upon request). Spearman correlations between quantitative PCR and Affymetrix were: leptin $r=0.82$, adiponectin $r=0.71$, LIPE $r=0.58$ and MCP1 $r=0.94$ (all $p<0.002$ ).

The diameter of fat cells was measured under a light microscope from fresh biopsies treated with collagenase. Fat-cell volume and weight were calculated assuming the adipocytes to be spheres and the density of human triacylglycerol to be $0.915 \mathrm{~g} / \mathrm{ml}$ [34]. The total number of fat cells in an individual was obtained by dividing the DXA-derived total body fat $(\mathrm{kg})$ by the mean weight of a single cell.

Diet, alcohol intake, smoking and physical activity Dietary intake was assessed from 3-day food records and analysed by the Diet32 program (Aivo), based on a national Finnish database for food composition (Fineli, www.fineli.fi, National Institute for Health and Welfare, Nutrition Unit, Helsinki, Finland). Information on smoking and weekly alcohol intake during the past 4 weeks was obtained by structured questionnaires and total physical activity by the Baecke questionnaire [35].

Statistical analyses Statistical analyses were performed with Stata statistical software (release 11.0; Stata Corporation, College Station, TX, USA). Results are expressed as mean \pm SE 
unless stated otherwise. Comparisons between the co-twins were made by matched pairs Wilcoxon's rank sum tests. Within-pair differences $(\Delta)$ were calculated by subtracting the leaner co-twin's value from the heavier co-twin's value. Spearman's correlations of the within-pair differences in all MZ twins were calculated to assess the relationships of the measures adjusted for genetic influences. Within-pair differences between the two groups of obesity-discordant MZ pairs, those who were discordant and those who were concordant for liver fat, identified based on clear-cut differences visualised in Fig. 1, were compared using the Mann-Whitney $U$ test. Group 1 denotes obesity-discordant co-twins that are metabolically similar, and group 2 denotes obesity-discordant cotwins where the obese co-twin has high liver fat and metabolic disturbances. The Mann-Whitney $U$ test was used to compare differences between lean participants in group 1 vs group 2, as well as obese participants in group 1 vs group 2 . Sex distributions between the groups were tested by the $\chi^{2}$ test. Because women had higher circulating and adipose tissue transcript levels of leptin than men, analyses on leptin were performed with sex-adjusted values. Multiple regression analyses were performed to detect independent predictors of $\Delta$ liver fat.

GO enrichment analysis was used to compute hypothesisfree enrichment of GO terms (molecular function) in each of the groups (weight-concordant, group 1 discordant and group 2 discordant). Enrichment analysis was done using Fisher's Exact Test. Fisher's test compares the observed frequency of each present GO term to the frequency in a reference gene set (whole gene array). A GO term is present if some input gene is annotated with the GO term or its descendants. The $p$ values are adjusted using the Benjamini and Hochberg FDR. The GO enrichment analysis was performed using the Anduril data analysis framework [36] and $\mathrm{R}$ [37].

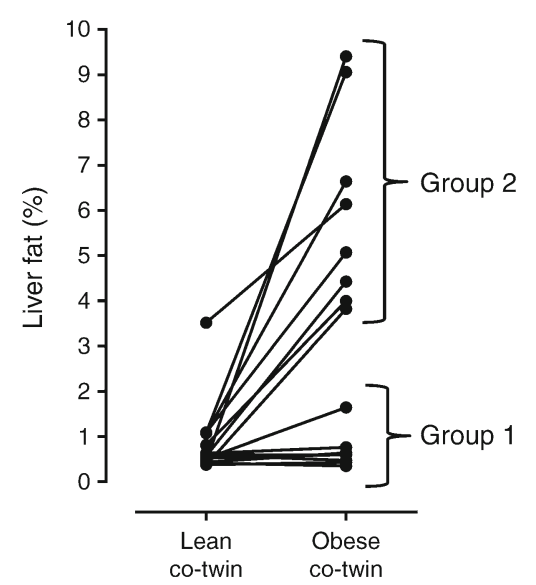

Fig. 1 Liver fat content determined by MRS in monozygotic obesitydiscordant pairs in group 1 with low $(<2 \%[n=8])$ and group 2 with high $(\geq 2 \%[n=8])$ within-pair differences in liver fat

\section{Results}

Two distinct groups of obesity-discordant MZ pairs The obesity-discordant pairs had a mean $\Delta$ weight of $17.4 \mathrm{~kg}$ (24\%) and large differences in all adiposity measures (Table 1). However, for liver fat two very distinct subgroups emerged within the obesity-discordant pairs (Fig. 1). In half of these pairs, the obese co-twins had liver fat percentages as low as those of their leaner co-twins ( $\Delta$ liver fat $8 \%$, Wilcoxon's test between co-twins $p=0.21$, group 1 , Fig. $1, n=8$ pairs), whereas in the other half, the obese co-twins had strikingly increased liver fat content ( $\Delta$ liver fat $718 \%, p=0.012$, group 2, Fig. 1, $n=8$ pairs).

Obesity-discordant pairs concordant and discordant for liver fat (Fig. 2a) did not differ for measures of overall fatness. Both groups had similar $\Delta$ weight (group 1, mean $17.4 \mathrm{~kg}$, range 11.1-30.3 kg; group 2, mean $17.1 \mathrm{~kg}$, range $9.6-25.8 \mathrm{~kg}$ ) and $\triangle \mathrm{BMI}$ (group 1, mean $5.9 \mathrm{~kg} / \mathrm{m}^{2}$; range $3.8-10.0 \mathrm{~kg} / \mathrm{m}^{2}$; group 2 , mean $5.5 \mathrm{~kg} / \mathrm{m}^{2}$, range $3.1-8.3 \mathrm{~kg} / \mathrm{m}^{2}$ ). Neither did the groups differ for $\triangle \mathrm{SAT}$ (Fig. 2b). However, $\triangle$ VAT was larger in group 2 than in group 1 pairs (Fig. 2c). Examples of twin pairs in each of the groups are presented in ESM Fig. 1.

Glucose and lipid metabolism and blood pressure In group 1, there were no differences between the obese and lean co-twins in glucose and insulin curves during the OGTT (Fig. 3). The co-twins also had similar insulin resistance (HOMA-IR, Fig. 2d) and insulin sensitivity (Matsuda, Fig. 2e). In group 2 , the obese co-twins had a significantly higher AUC for glucose $(23 \%, p=0.028)$ and insulin $(78 \%, p=0.028)$ during the OGTT (Fig. 3), as well as a $119 \%(p=0.018)$ higher HOMA-IR and 55\% $(p=0.028)$ lower Matsuda index than their lean co-twins (Fig. 2d, e). The obese co-twins in group 2 had significantly higher LDL- and lower HDL-cholesterol than their leaner co-twins (Table 1), while in group 1 the circulating lipids did not differ between co-twins. The blood pressure of the co-twins in both groups were similar, but a trend for increased blood pressure was observed in obese cotwins in group 2 (Table 1).

The obese co-twins in group 2 had significantly more liver fat $(p=0.0008)$ and VAT $(p=0.021)$, lower Matsuda index (Mann-Whitney $U$ test $p=0.006$ ) and higher HOMA-IR $(p=0.049)$ than the obese co-twins in group 1. Lean co-twins in group 2 did not differ from lean co-twins in group 1 in any of the metabolic values. Furthermore, co-twins from the weight-concordant group had similar metabolic measures (Table 1).

SAT cellularity and gene expression Compared with the leaner co-twins, the obese co-twins had $11 \%$ more adipocytes in group $1(p=0.069)$ but $8 \%$ less in group $2(p=0.13)$ and, accordingly, $\Delta$ adipocyte cell number differed significantly between the groups $(p=0.037)$. The mean adipocyte cell size 

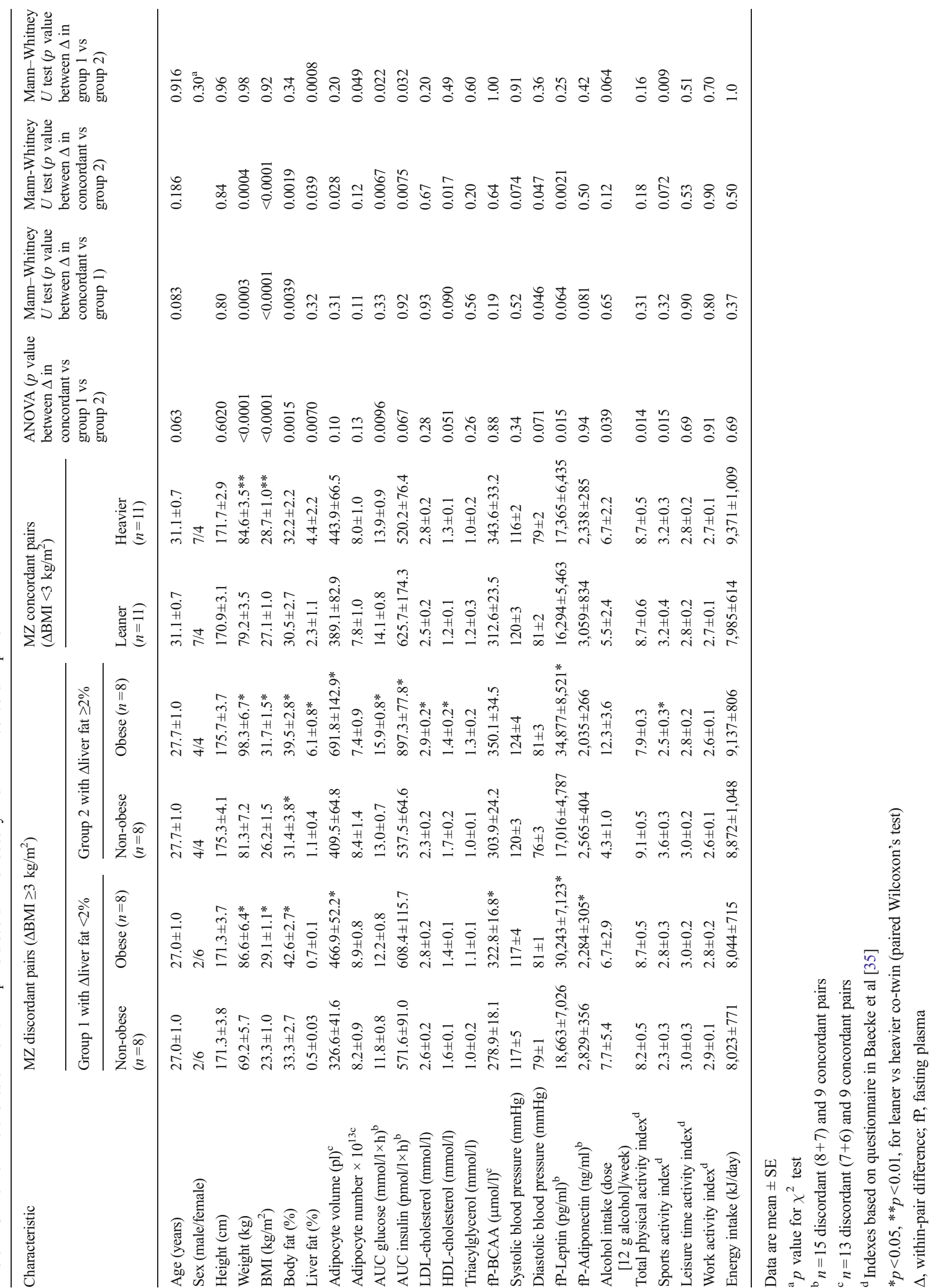
a
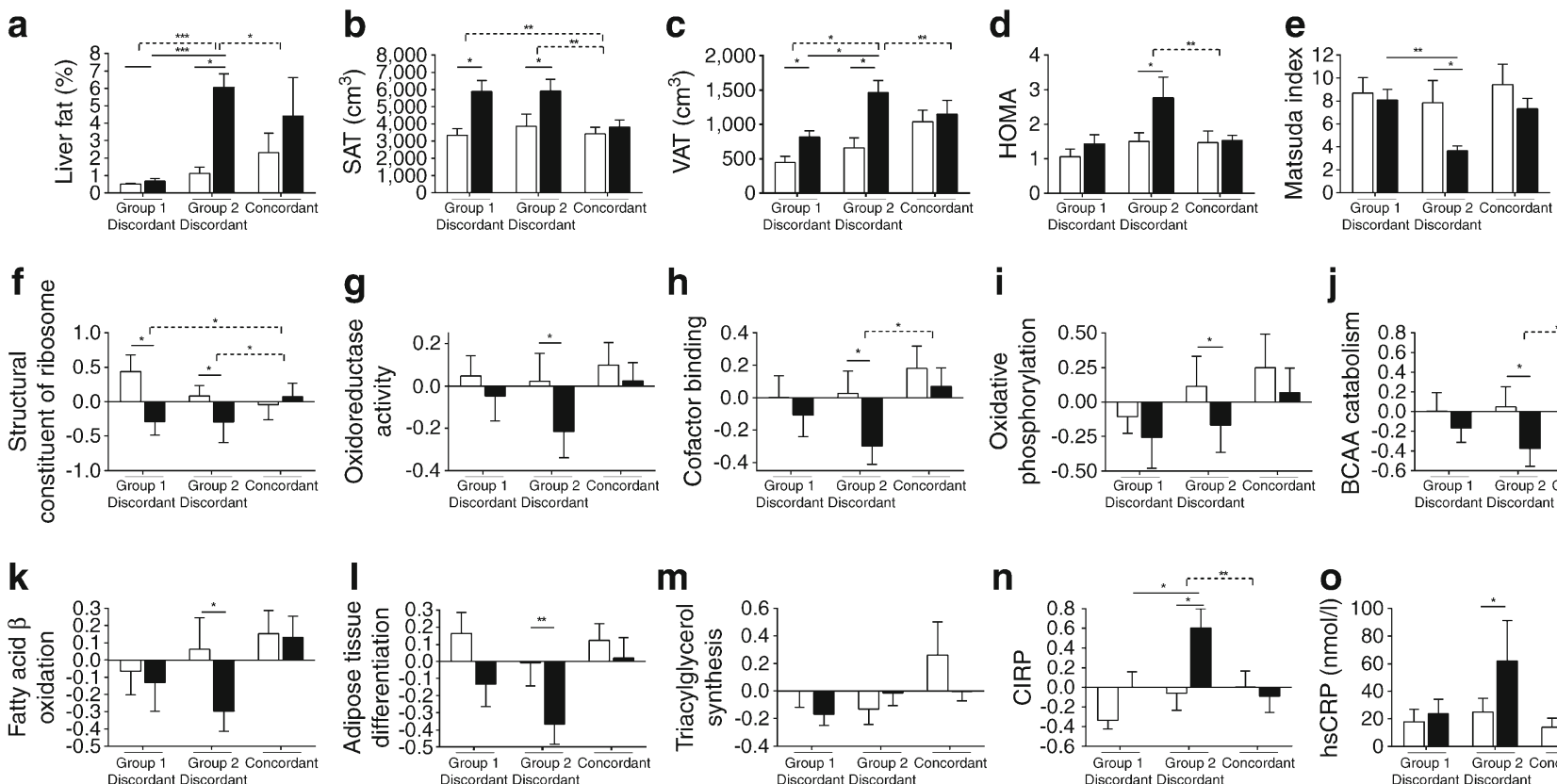

h

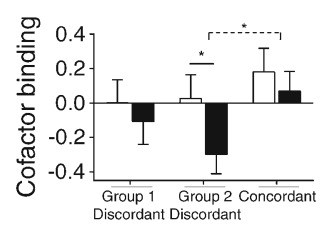

i
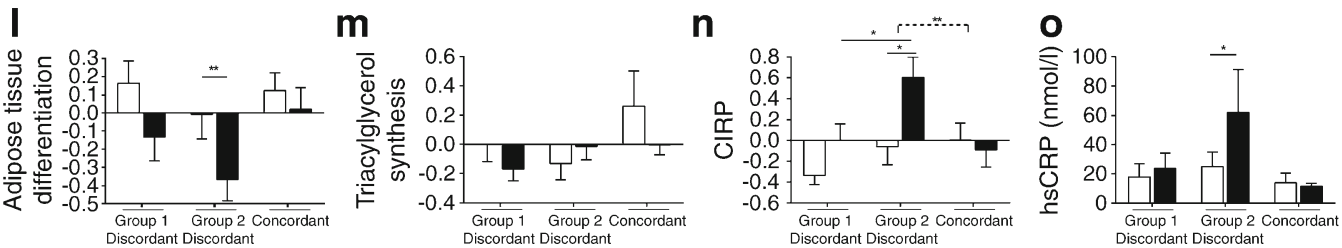

Fig. 2 Comparison of obesity-discordant pairs in group 1 with low $(<2 \%[n=8])$ vs group 2 with high $(\geq 2 \%[n=8])$ within-pair differences in liver fat. White bars, non-obese co-twins; black bars, obese co-twins.
Data are means \pm SE. ${ }^{*} p<0.05,{ }^{* *} p<0.01$ (matched pairs Wilcoxon's test within twin pairs and Mann-Whitney's $U$ test between groups) a
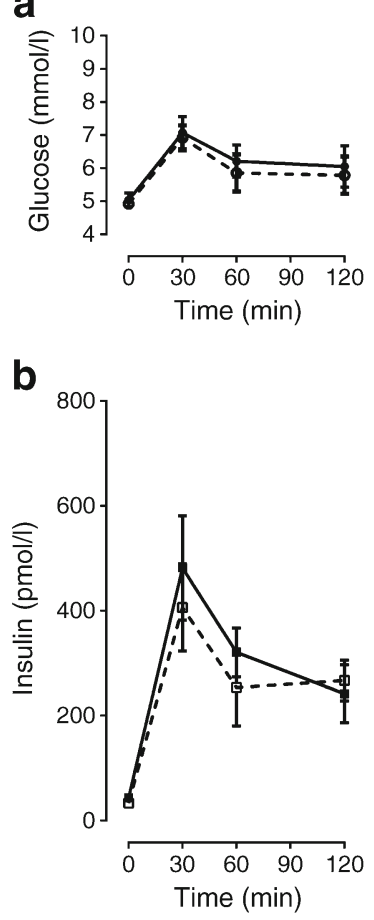

Group 1

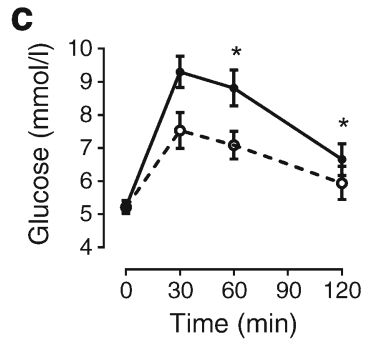

d

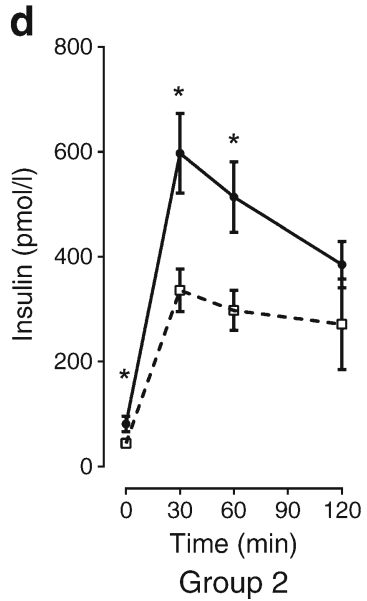

Fig. 3 Plasma glucose and serum insulin concentrations in the $2 \mathrm{~h}$ OGTT in obesity-discordant pairs in group 1 with low $(<2 \%[n=8])$ and group 2 with high $(\geq 2 \%[n=7])$ within-pair differences in liver fat. Note that of the original eight pairs in group 2 one pair in which the obese co-twin had type 2 diabetes was excluded. White symbols and dashed lines, nonobese co-twins; black symbols and solid lines, obese co-twins. Data are means \pm SE. ${ }^{*} p<0.05$ (matched pairs Wilcoxon's test within twin pairs)

was increased in all obese co-twins $(53 \%, p=0.017$ in group 1 and $69 \%, p=0.018$ in group 2) (Table 1).

Initially, a hypothesis-free $\mathrm{GO}$ enrichment analysis was done using the 'Molecular function' category to identify significant pathways. No differences were identified in the group of weight-concordant twins. Group 1 discordant twins differed only for 'structural constituent of ribosome' (GO:0003735) (FDR corrected $p=0.016$ ). Next, a mean centroid of this pathway was constructed, revealing a decreased transcriptional activity in every obese co-twin (also in group 2, although not reaching statistical significance) (Fig. 2f). In contrast, the group 2 obese and lean co-twins differed significantly for mitochondrial pathways in the obese co-twin, namely 'oxidoreductase activity' (GO:0016491) (FDR corrected $p=0.045$ ) and 'Cofactor binding' (GO:0048037) ( $p=0.0096)$, the latter of which also included several mitochondrial genes. These pathway activities were lower in the obese co-twins and restricted to group 2 (Fig. 2g, h).

Based on these findings, additional pathways of interest were studied to explain biological differences between groups 1 and 2. Three mitochondrial pathways were selected to represent different aspects of energy handling. All of them were downregulated in the SAT of group 2, but not group 1 obese co-twins: oxidative phosphorylation pathway ( $p=0.48$ in group 1, $p=0.028$ in group 2) (Fig. 2i); BCAA catabolism pathway ( $p=0.48$ in group $1, p=0.018$ in group 2) (Fig. $2 \mathrm{j}$ ) and fatty acid $\beta$ oxidation pathway ( $p=1.0$ in group1, $p=$ 0.018 in group 2) (Fig. 2k). Along with decreased catabolism, or clearance of BCAAs, circulating levels of BCAAs were 
increased in the obese co-twins compared with their lean counterparts $(p=0.016)$, but in within-group analyses the difference was statistically significant only in group 1 twins (Table 1).

We also tested two pathways relating to SAT enlargement (i.e. triacylglycerol synthesis and adipocyte cell differentiation). The triacylglycerol synthesis pathways did not differ between the obese and lean co-twins' SAT in either of the groups (Fig. $2 \mathrm{~m}$ ). In contrast, the adipocyte differentiation pathway was significantly downregulated in group 2 obese co-twins $(p=0.004)$, whereas the pathway expressions in group 1 co-twins were similar $(p=0.33)$ (Fig. 21). Further, the major transcript responsible for SAT lipolysis, LIPE, suggested that lipolysis was downregulated in group 2 but not in group 1 obese co-twins (ESM Fig. 2a).

Finally, significantly elevated activity of the chronic inflammatory response pathway (CIRP) was observed in the obese co-twins of group $2(p=0.028)$ but not group $1(p=0.31)$ (Fig. 2n). The CIRP pathway was also significantly more active in the obese co-twins from group 2 when compared with the equally obese co-twins from group $1(p=0.037)$ (Fig. 2n). Circulating hsCRP levels also differed between the co-twins only in group 2 (Fig. 2o).

Adipokines in serum and in SAT In both groups 1 and 2, the obese co-twins had significantly higher SAT expression and plasma levels of leptin than the non-obese co-twins (Table 1 and ESM Fig. 2b). Comparisons of group 1 and 2 were performed on sex-adjusted values, because both circulating $(p<0.001)$ and SAT leptin mRNA levels $(p=0.0095)$ were higher in women than in men. Despite similar BMI differences, plasma leptin concentrations showed larger intra-pair differences in group 2 than in group $1(p=0.014)$ suggesting that circulating leptin levels were disproportionally increased in the obese twins with adipose tissue inflammation and fatty livers. The obese co-twins had lower expression levels of adiponectin in adipose tissue only in group 2 ( $p=0.018$ vs $p=0.58$ in group 1) (ESM Fig. 2c). In plasma, $\Delta$ adiponectin was similar in both groups, but within-pair differences were statistically significant only in group 1 (Table 1 ).

Age, sex, lifestyle factors and onset of obesity Groups 1 and 2 were similar with regards to age, sex, smoking habits, alcohol intake, total physical activity and mean daily energy intake (Table 1). In group 2, sports activity was lower and alcohol intake tended to be higher in the obese than in lean co-twins. The onset of obesity discordance between the two groups was similar (18.8 and 20.0 years in groups 1 and 2, respectively).

Independent associations of adipose tissue function and liver fat $\Delta$ Liver fat correlated with $\Delta$ VAT $(r=0.40, p=0.038)$ but not with $\triangle$ SAT $(r=0.17, p=0.39)$. Of the metabolic measures studied, $\Delta$ liver fat correlated with $\triangle$ CIRP pathway $(r=0.44$, $p=0.026)$ and $\Delta$ plasma leptin $(r=0.51, p=0.011)$. In a multivariate model including both $\triangle \mathrm{VAT}$ and $\triangle \mathrm{SAT}$ and confounding factors (twin-pair sex, age and $\Delta$ sports activity), $\triangle$ VAT was the only variable independently explaining $\Delta$ liver fat $\left(\beta=4,800 \pm 1,600 \mathrm{~cm}^{3}, p=0.007\right.$, adjusted $R^{2}$ in the whole model $31 \%, p=0.023)$. However, when we included the two metabolic measures of SAT that were in univariate analyses associated with $\Delta$ liver fat $(\triangle \mathrm{CIRP}$ pathway expression and $\Delta$ plasma leptin), the results changed completely. The independent contributors to $\Delta$ liver fat in the subsequent model were $\triangle$ CIRP $(\beta=2.4 \pm 1.0, p=0.025), \Delta$ leptin $(\beta=0.00017 \pm$ $0.000056 \mathrm{pg} / \mathrm{ml}, p=0.008)$, age $(\beta=0.29 \pm 0.12$ years, $p=$ $0.030)$ and reduced $\Delta$ SAT $\left(\beta=-1,600 \pm 600 \mathrm{~cm}^{3}, p=0.017\right)$. The whole model explained $48 \%$ of variation in $\Delta$ liver fat $(p=0.019)$.

\section{Discussion}

With in-depth phenotyping of the exceptional obesitydiscordant young, healthy adult MZ twin pairs, it became evident that the metabolic responses to obesity differ vastly: half of the obese co-twins exhibited a typical response to obesity, with marked insulin resistance, dyslipidaemia and fatty liver (group 2), whereas the other half of the obese cotwins were metabolically as healthy as their lean co-twins (group 1). In the MHO group the obese and lean co-twins had remarkably similar profiles in numerous measures. Compared with their lean co-twins, despite an average of $17.4 \mathrm{~kg}$ higher body weight, the obese co-twins had identical liver fat levels, serum lipid profiles, responses to OGTT, blood pressure and levels of mitochondrial and inflammatory transcript pathways in SAT. In the other group of twin pairs, where the obese state accumulated fat in the liver, all of these measures were clearly disturbed in the obese as compared with lean co-twins.

Earlier studies have already established the accumulation of liver fat as being clearly linked to a disadvantageous metabolic phenotype $[12,14,15]$, in particular to insulin resistance $[14,15]$. In the present study, the most pronounced alteration in obese co-twins with high liver fat was the increase in insulin and glucose AUC during the OGTT. We also observed dyslipidaemia within these obese co-twins. All metabolic variables in our study of these young, still healthy, but obese individuals, were within the normal range, which suggests that the increase in liver fat is an early preclinical sign of metabolic disturbances. This was true even though not all obese co-twins exceeded the $5 \%$ increase generally considered pathological.

Our study demonstrated that excess fat accumulation in the liver varies among obese individuals. High liver fat can be observed in normal-weight individuals and even in conditions of lipodystrophy where SAT stores are depleted [38]. The 
mechanisms behind this mismatch are not completely understood. One hypothesis is the inadequate expandability of SAT, which directs lipids into ectopic sites [17]. Decreased lipid storage in SAT has previously been associated with accumulation of VAT in obesity [19] or during overfeeding [20]. Our study further suggests that deficient mitochondrial fat and amino acid oxidation in SAT may be associated with increased liver fat accumulation. Downregulation of mitochondrial metabolism reduces the availability of acetyl-CoA and ATP, key substrates necessary to support de novo lipogenesis [23] and lipolysis [39] within the adipocytes. Mitochondria are thus essential for flexible remodelling of SAT. Our samples were taken in a fasting condition, when no lipogenesis, but rather lipolysis, of SAT is expected. In line with this, the triacylglycerol synthesis pathway was not affected in either of the groups in obesity; in contrast, low LIPE mRNA expression suggested impaired lipolysis in group 2 obese twins. Downregulation of SAT lipolysis may also reflect preferential usage of hepatic lipids in individuals with high liver fat [40].

As multiple mitochondrial pathways are vital in the adipocyte differentiation process [41], it is also possible that mitochondrial malfunction impairs the development of new fat cells. This hypothesis was supported by a significantly decreased expression of the adipocyte differentiation pathway and a relative decrease in adipocyte number in group 2 obese co-twins. One possible mechanism behind impaired adipocyte differentiation could be decreased catabolism of BCAAs. Mitochondrial metabolism of BCAAs, especially leucine, stimulates adipocyte growth and differentiation through activation of the mammalian target of rapamycin (mTOR) signalling [42]. Our previous finding of downregulation of SAT BCAA catabolism transcription in obesity [24] was now shown to be restricted to only the group 2 obese co-twins. Decreased adipose BCAA catabolism, or clearance, would lead to a rise in plasma BCAA in obesity [43], a phenomenon first observed in the 1960s [44] and later found to predict subsequent development of type 2 diabetes [45].

One of the suspected hallmarks of MHO is a favourable plasma inflammation profile [4]. VAT but not SAT macrophage infiltration was found to distinguish insulin-resistant from insulin-sensitive morbidly obese individuals [10]. On the other hand, levels of SAT inflammatory mRNA markers and lipids were higher in obese women with high liver fat than in equally obese women with normal liver fat content [26]. In the present study, inflammation in both SAT and the circulation characterised metabolically deranged obesity. SAT inflammation, together with hyperleptinaemia and a relative decrease in SAT amount explained liver fat in the multivariate analyses within the twin pairs. Consistent findings of mitochondrial dysfunction and inflammation in SAT in the present and previous studies $[24,46]$ suggest that these two aberrations may produce a self-perpetuating vicious cycle. Whereas poor mitochondrial function and ATP production may violate the cells and direct them to phagocytosis, inflamed SAT may present a toxic environment for mitochondria and thus further risk the vitality of the tissue.

In rodents, knocking out genes that are involved in the formation of the inflammatory response prevent the development of type 2 diabetes [47-49] and in humans, use of antiinflammatory medications improves glycaemic control in type 2 diabetes and obesity [50]. In humans, the contribution of genes to the large metabolic variation in response to obesity is not known. Based on the present study, genetic factors determining mitochondrial function and inflammatory reactions may play a role in MHO. We also found some evidence for the role of lifestyle in MHO: the obese twins with metabolic aberrations had lower sports activity and a tendency for higher alcohol use than their lean co-twins but this pattern was not found in the MHO twins.

While this study has several advantages, it also has limitations. Young adult obesity-discordant MZ twins with no major confounding factors, such as medications and diseases, are extremely rare and, despite screening of ten yearly cohorts with well over 5,000 twin pairs, the final sample size was quite small. We did not have biopsies of VAT and liver and had SAT only from the periumbilical location, and thus we were not able to study the tissue-specific metabolism behind the group differences. The gene expression analyses were obtained from whole adipose tissue and contain the stromavascular cells, the independent contribution of which cannot be differentiated from that of the adipocytes. Transcript measurements of relevant genes may not represent functional protein levels. The cross-sectional nature of the study precludes the ability to solidly make cause-and-effect claims. It is also possible that the MHO stage will change with age or with advanced obesity. However, at present the two metabolically distinct groups were of the same age and had similar age of onset of obesity discordance. Weight differences between the groups were similar, but a given weight difference may have different metabolic effects depending on where in the distribution of BMI a pair is located.

In conclusion, in our collection of $\mathrm{MZ}$ twin pairs discordant for obesity, the SAT transcript profile in MHO was characterised by the maintenance of mitochondrial function and absence of inflammation. Only those individuals that, along with increased obesity, developed SAT dysfunction (low mitochondrial and high inflammatory transcript activity) had fatty livers and the hallmarks of the metabolic syndrome, impaired glucose tolerance and dyslipidaemia. The adipose tissue of the MHO individuals was characterised by the capability for both hypertrophy and hyperplasia of adipocytes with increasing BMI. Future studies of the MHO phenotype may suggest new potentially druggable targets - with the most proximal intervention point perhaps being improving mitochondrial function and prevention of inflammation in adipose tissue. 
Acknowledgements We thank the participants for their invaluable contributions to the study. M. Turunen, Helsinki University Central Hospital and L. Mustelin, University of Helsinki are acknowledged for their help in the collection of the data. E. Rautaheimo and E. Järvinen, University of Helsinki are thanked for their RT-PCR work. R. J. Rose, University of Indiana, is thanked for contributing to funding of FinnTwin16 and FinnTwin12.

Array data Microarray data are available in the ArrayExpress database (www.ebi.ac.uk/arrayexpress) under accession number E-MTAB-1895.

Funding The study was supported by grants from Helsinki University Central Hospital (AR, KH, NL), Novo Nordisk, Biomedicum Helsinki, Jalmari and Rauha Ahokas (KHP) and Finnish Medical Foundations (PP), Finnish Foundation for Cardiovascular Research (KHP), Competitive Research Funding of the Pirkanmaa Hospital District (EM) and the Academy of Finland Centre of Excellence in Complex Disease Genetics (JK). Data collection in FinnTwin16 and FinnTwin12 was supported by the National Institute of Alcohol Abuse and Alcoholism (grants AA-12502 and AA-09203 to R. J. Rose), Academy of Finland (grants 44069, 205585, 118555 to JK), EU funded projects TORNADO (FP7-KBBE-22270) and ENGAGE (FP7- HEALTH-F4-2007- 201413).

Duality of interest The authors declare that there is no duality of interest associated with this manuscript.

Contribution statement $\mathrm{KHP}, \mathrm{ARi}, \mathrm{JK}$ and JN conceived the study concept and design. KHP, ARi and JK were responsible for acquisition of data and funding. KHP examined the twins clinically and was responsible for carrying out the study protocol and statistical analyses. AH, JL and NL researched MRI and MRS data, SHe and ARo helped with handling of the fat biopsies, $\mathrm{TH}$ and $\mathrm{MO}$ performed BCAA measurements and $\mathrm{KV}$ and EM performed adiponectin and leptin measurements. LS and SHa were responsible for the GO enrichment analysis. SHe contributed to the manuscript writing. JN researched and interpreted data (transcriptomics analyses) and together with KHP wrote the manuscript. AS, PP and GF participated in interpretation of data. All authors contributed to discussion, edited the manuscript and approved the version to be published. KHP is the guarantor of this work and had full access to all of the data, and takes full responsibility for the integrity of the data and the accuracy of the data analysis.

\section{References}

1. Primeau V, Coderre L, Karelis AD et al (2010) Characterizing the profile of obese patients who are metabolically healthy. Int J Obes (2005) 35:971-981

2. Bluher M (2010) The distinction of metabolically 'healthy' from 'unhealthy' obese individuals. Curr Opin Lipidol 21:38-43

3. Wildman RP, Muntner P, Reynolds K et al (2008) The obese without cardiometabolic risk factor clustering and the normal weight with cardiometabolic risk factor clustering: prevalence and correlates of 2 phenotypes among the US population (NHANES 1999-2004). Arch Intern Med 168:1617-1624

4. Samocha-Bonet D, Chisholm DJ, Tonks K, Campbell LV, Greenfield JR (2012) Insulin-sensitive obesity in humans - a 'favorable fat' phenotype? Trends Endocrinol Metab 23:116-124

5. Jennings CL, Lambert EV, Collins M, Joffe Y, Levitt NS, Goedecke JH (2008) Determinants of insulin-resistant phenotypes in normal-weight and obese Black African women. Obesity (Silver Spring) 16:16021609

6. Messier V, Karelis AD, Prud'homme D, Primeau V, Brochu M, Rabasa-Lhoret R (2010) Identifying metabolically healthy but obese individuals in sedentary postmenopausal women. Obesity (Silver Spring) 18:911-917

7. Weiss R, Taksali SE, Dufour S et al (2005) The "obese insulinsensitive" adolescent: importance of adiponectin and lipid partitioning. J Clin Endocrinol Metabol 90:3731-3737

8. Brochu M, Tchernof A, Dionne IJ et al (2001) What are the physical characteristics associated with a normal metabolic profile despite a high level of obesity in postmenopausal women? J Clin Endocrinol Metabol 86:1020-1025

9. Karelis AD, Faraj M, Bastard JP et al (2005) The metabolically healthy but obese individual presents a favorable inflammation profile. J Clin Endocrinol Metabol 90:4145-4150

10. Kloting N, Fasshauer M, Dietrich A et al (2010) Insulin-sensitive obesity. Am J Physiol 299:E506-E515

11. Messier V, Karelis AD, Robillard ME et al (2010) Metabolically healthy but obese individuals: relationship with hepatic enzymes. Metab Clin Exp 59:20-24

12. Stefan N, Kantartzis K, Machann J et al (2008) Identification and characterization of metabolically benign obesity in humans. Arch Intern Med 168:1609-1616

13. Coen PM, Dube JJ, Amati F et al (2010) Insulin resistance is associated with higher intramyocellular triglycerides in type I but not type II myocytes concomitant with higher ceramide content. Diabetes 59:80-88

14. Fabbrini E, Magkos F, Mohammed BS et al (2009) Intrahepatic fat, not visceral fat, is linked with metabolic complications of obesity. Proc Natl Acad Sci U S A 106:15430-15435

15. D'Adamo E, Cali AM, Weiss R et al (2010) Central role of fatty liver in the pathogenesis of insulin resistance in obese adolescents. Diabetes Care 33:1817-1822

16. Bedogni G, Miglioli L, Masutti F, Tiribelli C, Marchesini G, Bellentani S (2005) Prevalence of and risk factors for nonalcoholic fatty liver disease: the Dionysos nutrition and liver study. Hepatology (Baltimore) 42:44-52

17. Heilbronn L, Smith SR, Ravussin E (2004) Failure of fat cell proliferation, mitochondrial function and fat oxidation results in ectopic fat storage, insulin resistance and type II diabetes mellitus. Int J Obes Relat Metab Disord 28(Suppl 4):S12-S21

18. Capeau J, Magre J, Lascols O et al (2005) Diseases of adipose tissue: genetic and acquired lipodystrophies. Biochem Soc Trans 33:1073-1077

19. McQuaid SE, Hodson L, Neville MJ et al (2011) Downregulation of adipose tissue fatty acid trafficking in obesity: a driver for ectopic fat deposition? Diabetes 60:47-55

20. Alligier M, Gabert L, Meugnier E et al (2013) Visceral fat accumulation during lipid overfeeding is related to subcutaneous adipose tissue characteristics in healthy men. J Clin Endocrinol Metabol 98:802-810

21. De Pauw A, Tejerina S, Raes M, Keijer J, Arnould T (2009) Mitochondrial (dys)function in adipocyte (de)differentiation and systemic metabolic alterations. Am J Pathol 175:927-939

22. Kaaman M, Sparks LM, van Harmelen V et al (2007) Strong association between mitochondrial DNA copy number and lipogenesis in human white adipose tissue. Diabetologia 50:2526-2533

23. Medina-Gomez G (2012) Mitochondria and endocrine function of adipose tissue. Best Pract Res 26:791-804

24. Pietiläinen KH, Naukkarinen J, Rissanen A et al (2008) Global transcript profiles of fat in monozygotic twins discordant for BMI: pathways behind acquired obesity. PLoS Med 5:e51

25. Mustelin L, Pietiläinen KH, Rissanen A et al (2008) Acquired obesity and poor physical fitness impair expression of genes of mitochondrial oxidative phosphorylation in monozygotic twins discordant for obesity. Am J Physiol 295:E148-E154

26. Kolak M, Westerbacka J, Velagapudi VR et al (2007) Adipose tissue inflammation and increased ceramide content characterize subjects with high liver fat content independent of obesity. Diabetes 56:1960-1968

27. Naukkarinen J, Rissanen A, Kaprio J, Pietiläinen KH (2012) Causes and consequences of obesity: the contribution of recent twin studies. Int J Obes (London) 36:1017-1024 
28. Kaprio J (2006) Twin studies in Finland 2006. Twin Res Hum Genet 9:772-777

29. Graner M, Seppälä-Lindroos A, Rissanen A et al (2012) Epicardial fat, cardiac dimensions, and low-grade inflammation in young adult monozygotic twins discordant for obesity. Am J Cardiol 109:12951302

30. Matthews DR, Hosker JP, Rudenski AS, Naylor BA, Treacher DF, Turner RC (1985) Homeostasis model assessment: insulin resistance and beta-cell function from fasting plasma glucose and insulin concentrations in man. Diabetologia 28:412-419

31. Matsuda M, DeFronzo RA (1999) Insulin sensitivity indices obtained from oral glucose tolerance testing: comparison with the euglycemic insulin clamp. Diabetes Care 22:1462-1470

32. Yeckel CW, Weiss R, Dziura J et al (2004) Validation of insulin sensitivity indices from oral glucose tolerance test parameters in obese children and adolescents. J Clin Endocrinol Metabol 89: 1096-1101

33. Castillo S, Mattila I, Miettinen J, Oresic M, Hyotylainen T (2011) Data analysis tool for comprehensive two-dimensional gas chromatography/time-of-flight mass spectrometry. Anal Chem 83: 3058-3067

34. Hirsch J, Gallian E (1968) Methods for the determination of adipose cell size in man and animals. J Lipid Res 9:110-119

35. Baecke JA, Burema J, Frijters JE (1982) A short questionnaire for the measurement of habitual physical activity in epidemiological studies. Am J Clin Nutr 36:936-942

36. Ovaska K, Laakso M, Haapa-Paananen S et al (2010) Large-scale data integration framework provides a comprehensive view on glioblastoma multiforme. Genom Med 2:65

37. R Development Core Team (2010) R: a language and environment for statistical computing. R Foundation for Statistical Computing, Vienna

38. Agarwal AK, Garg A (2003) Congenital generalized lipodystrophy: significance of triglyceride biosynthetic pathways. Trends Endocrinol Metab 14:214-221
39. Fassina G, Dorigo P, Gaion RM (1974) Equilibrium between metabolic pathways producing energy: a key factor in regulating lipolysis. Pharmacol Res Commun 6:1-21

40. Sunny NE, Parks EJ, Browning JD, Burgess SC (2011) Excessive hepatic mitochondrial TCA cycle and gluconeogenesis in humans with nonalcoholic fatty liver disease. Cell Metab 14:804-810

41. Hackl H, Burkard TR, Sturn A et al (2005) Molecular processes during fat cell development revealed by gene expression profiling and functional annotation. Genome Biol 6:R108

42. Lynch CJ, Halle B, Fujii $\mathrm{H}$ et al (2003) Potential role of leucine metabolism in the leucine-signaling pathway involving mTOR. Am J Physiol 285:E854-E863

43. Herman MA, She P, Peroni OD, Lynch CJ, Kahn BB (2010) Adipose tissue branched chain amino acid (BCAA) metabolism modulates circulating BCAA levels. J Biol Chem 285:11348-11356

44. Felig P, Marliss E, Cahill GF Jr (1969) Plasma amino acid levels and insulin secretion in obesity. N Engl J Med 281:811-816

45. Wang TJ, Larson MG, Vasan RS et al (2011) Metabolite profiles and the risk of developing diabetes. Nat Med 17:448-453

46. Soronen J, Laurila PP, Naukkarinen J et al (2012) Adipose tissue gene expression analysis reveals changes in inflammatory, mitochondrial respiratory and lipid metabolic pathways in obese insulin-resistant subjects. BMC Med Genet 5:9

47. Nomiyama T, Perez-Tilve D, Ogawa D et al (2007) Osteopontin mediates obesity-induced adipose tissue macrophage infiltration and insulin resistance in mice. J Clin Invest 117:2877-2888

48. Arkan MC, Hevener AL, Greten FR et al (2005) IKK-beta links inflammation to obesity-induced insulin resistance. Nat Med 11:191-198

49. Uysal KT, Wiesbrock SM, Marino MW, Hotamisligil GS (1997) Protection from obesity-induced insulin resistance in mice lacking TNF-alpha function. Nature 389:610-614

50. Goldfine AB, Fonseca V, Jablonski KA, Pyle L, Staten MA, Shoelson SE (2010) The effects of salsalate on glycemic control in patients with type 2 diabetes: a randomized trial. Ann Intern Med $152: 346-357$ 